Pacific Journal of Mathematics

ABOUT COMPRESSIBLE VISCOUS FLUID FLOW IN A 


\section{ABOUT COMPRESSIBLE VISCOUS FLUID FLOW IN A BOUNDED REGION}

\section{Gerhard STRÖHMER}

This paper deals with the question of existence for all times of the solutions of a certain class of differential equations for small initial values, and with the asymptotic behavior of these solutions. This class of equations contains different models describing the flow of viscous compressible fluids, even under the influence of a magnetic field.

1. Introduction. We consider the initial-boundary value problem on a bounded domain $\Omega \subset \mathbb{R}^{n}$ with Dirichlet boundary conditions $\left(\partial \Omega \in C^{3}\right)$. The solutions of our equations are functions $X: \bar{\Omega} \times$ $[0, \infty) \rightarrow \mathbb{R}^{m+1}(X=X(x, t), m \geq n)$ representing the relevant physical variables in their dependence on space and time. For the sake of simplicity we assume that $\Omega_{1} \subset \mathbb{R}^{m+1}$ is a convex domain containing all physically reasonable values of $X$. The set might, e.g., include only positive values for density (which is usually the $(m+1)$ st component of $X$ ), and temperature. Then our equations have the form

$$
\begin{gathered}
X_{t}^{l}+f^{l}(X, \nabla X)=L_{X}^{l} X+g^{l}(x, t) \quad(l=1, \ldots, m), \\
X_{t}^{m+1}+\sum_{i=1}^{n}\left(X^{m+1} X^{i}\right)_{x_{t}}=0
\end{gathered}
$$

with sufficiently regular functions $f: \Omega_{1} \times \mathbb{R}^{n(m+1)} \rightarrow \mathbb{R}^{m}$ and $g: \Omega \times[0, \infty) \rightarrow \mathbb{R}^{m}$, and an elliptic operator $L_{Y} X$ given by

$$
L_{Y}^{l} X=\sum_{i=1}^{m} \sum_{j, k=1}^{n} a_{i}^{k j l}(Y) X_{x_{k} x_{j}}^{i} \quad(l=1, \ldots, m)
$$

with $a_{i}^{k j l} \in C^{3}\left(\Omega_{1}\right)$. The solutions we obtain are small in the sense of being close to a constant state $H_{\infty} \in \Omega_{1}$. The most important hypothesis we need for our result is expressed in Condition $\mathrm{C}$, which states roughly that the linearization of our equation at $H_{\infty}$ generates an analytic semigroup with exponential decay for $t \rightarrow \infty$ on a product of $L_{p}$-based Sobolev spaces for some $p>n$. This decay is the most important factor in the proof of a priori estimates for solutions of 
(E) in Chapter 2, and their differences in Chapter 3, which enable us to prove the existence of a solution of $E$ if $g$ is sufficiently small and the initial values are sufficiently close to $H_{\infty}$, and to describe its asymptotic behavior.

In contrast to the situation in one (see, e.g., [4]) and two space dimensions (in the latter case there are at least global weak solutions for isothermal gas flow in $\mathbb{R}^{2}$, as is shown in [8]), the only known results about existence for all times of solutions for the equations of viscous compressible fluid flow in higher dimensions impose smallness conditions on initial and boundary data, and the exterior forces. They were proved by Matsumura and Nishida ([5], [6], [7]) for domains with and without boundary. These papers also contain results about the asymptotic behavior of the solution; here a smallness condition for exterior forces and boundary values seems to be necessary, as turbulence is bound to occur at some point, preventing convergence as $t \rightarrow \infty$. For bounded domains our results go beyond those of Matsumura and Nishida. We give a rather general sufficient condition for the existence of a small solution for all times, we do not require the exterior forces to be conservative, nor the boundary values to be constant, and we do not need to confine ourselves to three dimensions. (For the incompressible case see, e.g., [12].)

Let us now discuss some examples of systems of equations accessible to our method. In [10] Condition $\mathrm{C}$ is verified for compressible, viscous, and heat conducting flow. The system of equations

$$
\begin{gathered}
\rho_{t}+\operatorname{div}(\rho u)=0, \\
u_{t}+(u \cdot \nabla) u+\frac{1}{\rho} \nabla(p(\rho, \theta)) \\
=\frac{\sigma}{\rho}(u \times B) \times B+\frac{1}{\rho}\left[\operatorname{div}(\mu \nabla u)+\nabla\left(\left(\mu^{\prime}+\mu\right) \operatorname{div} u\right)\right], \\
\theta_{t}+(u \cdot \nabla) \theta+\frac{\theta p_{\theta}(\rho, \theta)}{\rho c} \operatorname{div} u=\frac{1}{\rho c}(\operatorname{div}(k \nabla \theta)+\psi)
\end{gathered}
$$

with

$$
\psi=\frac{\mu}{2}\left(u_{x_{k}}^{j}+u_{x_{j}}^{k}\right)^{2}+\mu^{\prime}(\operatorname{div} u)^{2}+\sigma|u \times B|^{2},
$$

which describes the flow of an ionized gas with density $\rho$, temperature $\theta$, and velocity $u\left(\mu, \mu^{1}\right.$ viscosity coefficients, $c$ heat capacity, $\sigma$ electric conductivity, $p=p(\rho, \theta)$ pressure) under the influence of a magnetostatic field $B(x) \in C^{3}(\bar{\Omega})$, which is assumed not to be perceptibly influenced by the currents in the gas, is another example (see [2], [9]). (Note that $B$ is not required to be small.) The proof that 
this system fulfills $\mathrm{C}$ parallels [10] closely. For the analog of Lemma 3.5 use

$$
((u \times B) \times B) \cdot \bar{u}=(u \times B) \cdot(B \times \bar{u})=-(u \times B) \cdot(\overline{u \times B})=-|u \times B|^{2} .
$$

The magneto-fluid-dynamic system obtained by adding $B$ as an unknown function, and completing the system by the equation

$$
B_{t}=\sigma^{-1} \mu_{m}^{-1} \Delta B-\operatorname{curl}(u \times B)
$$

( $\mu_{m}$ magnetic permeability) also fulfills condition $\mathrm{C}$ if the part of $H_{\infty}$ corresponding to $B$ is zero. Dirichlet boundary conditions for $B$ seem, however, not very suited to this problem, as they usually interfere with the condition $\operatorname{div} B=0$.

Now we make our statements precise. (For the definitions of some of the objects mentioned here see 1.3.) We denote the two groups of variables of $f$ by $H$ and $P(f=f(H, P))$, with $H=\left(H^{1}, \ldots\right.$, $\left.H^{m+1}\right) \in \mathbb{R}^{m+1}, P=\left(P_{j}^{i}\right)_{i=1 j=1}^{m+1 n} \in \mathbb{R}^{n(m+1)}$. Then we suppose $f \in C^{3}$, $f\left(H_{\infty}, 0\right)=0, f_{H^{m+1}}\left(H_{\infty}, 0\right)=0$, and finally $\pi_{n} H_{\infty}=0$, which is reasonable if the first $n$ components of $X$ represent the velocity of the flow. We also assume the ellipticity condition

$$
\sum_{i=1}^{m} a_{i}^{k j l}(H) \eta_{i} \eta_{l} \xi_{k} \xi_{j}>0 \quad\left(H \in \Omega_{1}, \eta \in \mathbb{R}^{m}, \xi \in \mathbb{R}^{n}, \eta \neq 0 \neq \xi\right) .
$$

As a final step we make a number of definitions needed to state Condition C. For technical reasons the Sobolev spaces in the following section are complex, although our solutions are real. We first define the linear operator

$$
A_{1}:\left(H_{p}^{2}\right)^{m} \times H_{p}^{1} \rightarrow\left(L_{p}\right)^{m} \times H_{p}^{1}
$$

by

$$
\begin{array}{r}
\left(A_{1} X\right)^{l}=f_{H_{i}}^{l}\left(H_{\infty}, 0\right) X^{i}+\sum_{\mu=1}^{m} \sum_{\nu=1}^{n} f_{P_{\nu}^{\mu}}^{l}\left(H_{\infty}, 0\right) X_{y_{\nu}}^{\mu}-L_{H_{\infty}} X \\
(l=1, \ldots, m),
\end{array}
$$

and $\left(A_{1} X\right)^{m+1}=H_{\infty}^{m+1} \sum_{i=1}^{n} X_{x_{t}}^{i}$. Now we need two spaces defined in analogy to [10]:

$$
\begin{aligned}
& B_{p}=\left\{X: \Omega \rightarrow \mathbb{C}^{m+1} \mid X \in\left(L_{p}\right)^{m} \times H_{p}^{1}, \int_{\Omega} X^{m+1} d x=0\right\}, \\
& D_{A}^{p}=B_{p} \cap\left\{X \in\left(H_{p}^{2}\right)^{m} \times H_{p}^{1}\left|\pi_{m} X\right| \partial \Omega=0\right\} .
\end{aligned}
$$


These sets are Banach spaces with respect to any norm of $\left(L_{p}\right)^{m} \times H_{p}^{1}$ and $\left(H_{p}^{2}\right)^{m} \times H_{p}^{1}$, respectively. We choose one of these in each case and denote them by $\|\cdot\|_{B_{p}}$ and $\|\cdot\|_{D_{A}^{p}}$. Then let $A=A_{1} \mid D_{A}^{p}$; note that $A\left(D_{A}^{p}\right) \subset B_{p}$. This allows us to formulate

Condition C. The operator $A: D_{A}^{p} \rightarrow B_{p}$ is closed, and there are numbers $\eta>0$, and $K_{2}<\infty$ such that the resolvent $(A+z I)^{-1}$ of $A$ exists at least for all elements $z$ of

$$
\mathscr{R}=\left\{z \in \mathbb{C} \mid \operatorname{Re} z \geq-2 \eta, \text { or } \operatorname{Re} z \geq-|\operatorname{Im} z| \text { and }|z| \geq \eta^{-1}\right\},
$$

and

$$
\left\|(A+z I)^{-1} X\right\|_{B_{p}} \leq K_{2}(1+|z|)^{-1}\|X\|_{B_{p}}
$$

for all $X \in B_{p}, z \in \mathscr{R}$. In addition,

$$
\begin{gathered}
\|X\|_{D_{p}} \leq K_{2}\|(A+z I) X\|_{B_{p}}, \\
\|X\|_{\left(H_{p}^{1}\right)^{m} \times H_{p}^{2}} \leq K_{2}(1+|z|)^{-1 / 2}\|(A+z I) X\|_{\left(H_{p}^{1}\right)^{m} \times H_{p}^{2}}
\end{gathered}
$$

and

$$
\|X\|_{\left(H_{p}^{3}\right)^{m} \times H_{p}^{2}} \leq K_{2}(1+|z|)^{1 / 2}\|(A+z I) X\|_{\left(H_{p}^{1}\right)^{m} \times H_{p}^{2}}
$$

for all $z \in \mathscr{R}, X \in D_{A}^{p}$, and $X \in\left(H_{p}^{3}\right)^{m} \times H_{p}^{2}$ in addition for the last two inequalities. Now we can state our two theorems.

1.1. ThEOREM. Assume the system of equations (E) together with $H_{\infty} \in \Omega_{1}$ fulfill Condition $\mathrm{C}$, and the other hypotheses stated as yet. Then there are numbers $\varepsilon_{1}>0$, and $K_{1}<+\infty$ such that if $X_{0} \in H_{p}^{2}$, $(\beta, g) \in \mathscr{B}$ (see 1.3) with $\beta(0)=X_{0} \mid \partial \Omega, \pi_{n} \beta=0$, and

$$
\left\|X_{0}-H_{\infty}\right\|_{H_{p}^{2}}+\left\|\left(\beta-\pi_{m} H_{\infty}, g\right)\right\|_{\mathscr{B}} \leq \varepsilon_{1},
$$

then there is exactly one function $X=X(x, t)$ belonging to

$$
C^{0}\left([0, \infty), H_{p}^{2}\right) \cap C^{1}\left([0, \infty), L_{p}\right) \cap C^{1}\left((0, \infty), H_{p}^{1}\right)
$$

with $\pi_{m} X \in C^{0}\left((0, \infty), H_{p}^{3}\right)$, and solving (E) in the classical sense for $t>0$, such that $\pi_{m} X \mid \partial \Omega=\beta$, and $X(x, 0)=X_{0}(x)$ for $x \in \bar{\Omega}$. For $t \geq 1$ this function fulfills the inequality

$$
\begin{aligned}
& \|X(x, t)\|_{H_{p}^{2}}+\left\|\pi_{m} X(x, t)\right\|_{H_{p}^{3}} \\
& \quad \leq K_{1}\left(\left\|X_{0}-H_{\infty}\right\|_{H_{p}^{2}}+\left\|\left(\beta-\pi_{m} H_{\infty}, g\right)\right\|_{\mathscr{B}}\right) .
\end{aligned}
$$


1.2. THEOREM. There is an $\varepsilon_{2}>0$ such that if

$$
\left\|X_{0}-H_{\infty}\right\|_{H_{p}^{2}}+\left\|\left(\beta-\pi_{m} H_{\infty}, g\right)\right\|_{\mathscr{B}} \leq \varepsilon_{2}
$$

in addition to the assumptions of 1.1 , and $\beta(t) \rightarrow \hat{\beta}$ in $H_{p}^{2}(\partial \Omega)$, $\beta_{t} \rightarrow 0$ in $L_{p}(\partial \Omega)$, and $g(t) \rightarrow \hat{g}$ in $L_{p}(t \rightarrow \infty)$, then the solution $X(x, t)$ mentioned in 1.1 converges weakly in $H_{p}^{2}$ to a function $\widehat{X}$, and $\pi_{m} X(x, t)$ even converges weakly in $H_{p}^{3}$ as $t \rightarrow \infty$. Also $\hat{X}$ is a time-independent solution of $(\mathrm{E})$ with boundary values $\hat{\beta}$ and right side $\hat{g}$. It is the only such solution fulfilling the inequality

$$
\left\|\widehat{X}-H_{\infty}\right\|_{H_{p}^{2}}+\left\|\pi_{m}\left(\widehat{X}-H_{\infty}\right)\right\|_{H_{p}^{3}} \leq K_{1} \varepsilon_{2} .
$$

1.3. Notation. In the symbols used for function spaces the set on which the functions are defined is often omitted, this means usually that the set is $\Omega$, except in statements like $f \in C^{3}$, where $f$, and its domain, have been given before. In general we take over the notation of [11], with the exception that $X$ represents here what would have been $X+H_{\infty}$ there. Let

$$
\begin{array}{r}
\mathscr{B}=\left\{(\beta, g) \mid \beta: \partial \Omega \rightarrow \mathbb{R}^{m}, g: \bar{\Omega} \rightarrow \mathbb{R}^{m}, \beta \in C^{1}\left([0, \infty), H_{p}^{3}(\partial \Omega)\right)\right. \\
\cap C^{2}\left([0, \infty), H_{p}^{1}(\partial \Omega)\right), \text { and } \\
\left.\quad g \in C^{0}\left([0, \infty), H_{p}^{2}\right) \cap C^{1}\left([0, \infty), H_{p}^{1}\right)\right\},
\end{array}
$$

for $(\beta, g) \in \mathscr{B}$ we define

$$
\begin{aligned}
\|(\beta, g)\|_{\mathscr{B}}= & \|\beta\|_{C^{1}\left([0, \infty), H_{p}^{3}(\partial \Omega)\right)}+\|\beta\|_{C^{2}\left([0, \infty), H_{p}^{1}(\partial \Omega)\right)} \\
& +\|g\|_{C^{0}\left([0, \infty), H_{p}^{2}\right)}+\|g\|_{C^{1}\left([0, \infty), H_{p}^{1}\right)} .
\end{aligned}
$$

For $\mu \geq \nu$ let $\pi_{r}: \mathbb{R}^{\mu} \rightarrow \mathbb{R}^{r}$ be defined by $\pi\left(x_{1}, \ldots, x_{\mu}\right)=\left(x_{1}, \ldots\right.$, $\left.x_{\nu}\right)$. For $h: \Omega \rightarrow \mathbb{R}^{m+1}, h \in L_{1}$ let

$$
\begin{gathered}
M(h)=\left(0, \ldots, 0,|\Omega|^{-1} \int_{\Omega} h^{m+1} d x\right) \text { and } \\
\widetilde{H}_{\infty}(h)=\left(\pi_{m} H_{\infty}, M^{m+1}(h)\right) .
\end{gathered}
$$

All constants in this paper are independent of $T$ unless otherwise stated.

2. Existence of a global solution. We begin this chapter by stating an easy consequence of the local existence theorem proved in 
[11]. Then we show that every solution of $(\mathrm{E})$-expressed in Lagrange coordinates-solves the equation $X_{t}+A_{1} X=G(X)$ with the $G(X)$ defined in 2.2. We use this information to prove an a priori estimate which-together with the local existence result 2.1 -then gives the existence of a global solution.

2.1. Lemma. To every $T>0$ there is a $\delta_{1}>0$ such that if $(\beta, g) \in \mathscr{B}, X_{0} \in H_{p}^{2}, X_{0} \mid \partial \Omega=\beta(0), \pi_{n} \beta=0$, and

$$
\left\|\left(\beta-\pi_{m} H_{\infty}, g\right)\right\|_{\mathscr{B}}+\left\|X_{0}-H_{\infty}\right\|_{H_{p}^{2}} \leq \delta_{1},
$$

then there is exactly one function $X \in C^{0}\left([0, T], H_{p}^{2}\right)$ with $\pi_{m} X \in$ $C^{0}\left((0, T], H_{p}^{3}\right)$ which is a classical solution of $(\mathrm{E})$ for $t>0$ with $X(x, 0)=X_{0}(x)$ for $x \in \bar{\Omega}, X(x, t)=\beta(x, t)$ for $x \in \partial \Omega$, and $X$ fulfills the inequality

$$
\begin{aligned}
& \left\|\pi_{m}\left(X-H_{\infty}\right)\right\|_{H_{p}^{3}} \sqrt{t}+\left\|X-H_{\infty}\right\|_{H_{p}^{2}} \\
& \quad \leq K_{3}(T)\left(\left\|\left(\beta-\pi_{m} H_{\infty}, g\right)\right\|_{1}+\left\|X_{0}-H_{\infty}\right\|_{H_{p}^{2}}\right) .
\end{aligned}
$$

Proof. This can be obtained by repeated application of Theorem 1.1 and Theorem 5.2 of [11].

By Lemma 2.6 and Theorem 2.7 of [11], it is clear that $X(x, t)$ can be transformed into Lagrange coordinates in all of $[0, T]$ if

$$
\left\|\left(\beta-\pi_{m} H_{\infty}, g\right)\right\|_{\mathscr{B}}+\left\|X_{0}-H_{\infty}\right\|_{H_{p}^{2}}
$$

is small enough, and that the transformed solution $X(y, t)$ has the property

$$
\left\|X(y, t)-H_{\infty}\right\|_{\mathscr{S}(T)} \leq \widetilde{K}_{3}(T)\left(\left\|\left(\beta-\pi_{m} H_{\infty}, g\right)\right\|_{\mathscr{B}}+\left\|X_{0}-H_{\infty}\right\|_{H_{p}^{2}}\right)
$$

with a suitable constant $\widetilde{K}_{3}(T)$. (For the definition of $\mathscr{S}(T)$ see [11], Def. 3.1.)

The following definitions will be applied to such solutions.

2.2. Definition and Lemma. To every $T>0$ there is a $\delta_{2}>0$ such that if $X-H_{\infty} \in \mathscr{S}(T),\left\|X-H_{\infty}\right\|_{\mathscr{S}(T)} \leq \delta_{2}$, and $\pi_{n} X \mid \partial \Omega=0$, then

$$
\underline{T}_{t}^{X}(y)=y+\int_{0}^{t} \pi_{n} X(y, \tau) d \tau \quad(y \in \bar{\Omega})
$$


is an admissible family of transformations on $\bar{\Omega}$ (see Chap. 2 of [11]). With $x=\underline{T}_{t}^{X}(y)$ we then define $G(X)$ as follows: For $l=1, \ldots, m$ let

$$
\begin{aligned}
G^{l}(X)= & a_{i}^{k j l}(X(y, t)) X_{y_{\nu}}^{i}\left(\left(\underline{T}_{t}^{X}\right)^{-1}\right)_{x_{k} x_{j}}^{\nu}(y, t)+f_{H_{t}}^{l}\left(H_{\infty}, 0\right) X^{i} \\
& +\sum_{\nu=1}^{n} \sum_{\mu=1}^{m} f_{P_{\nu}^{\mu}}^{l}\left(H_{\infty}, 0\right) X_{y_{\nu}}^{\mu}-\bar{f}\left(X, X_{y_{\nu}}^{i}\left(\left(\underline{T}_{t}^{X}\right)^{-1}\right)_{x_{\mu}}^{\nu}\right) \\
& +\left[a_{i}^{k j l}(Y)\left(\left(\underline{T}_{t}^{X}\right)^{-1}\right)_{x_{k}}^{\nu}\left(\left(\underline{T}_{t}^{X}\right)^{-1}\right)_{x_{j}}^{\mu}-a_{l}^{\nu \mu l}\left(H_{\infty}\right)\right] X_{y_{\mu}}^{i} y_{\nu} \\
& +g\left(\underline{T}_{t}^{X}(y), t\right), \quad\left(\bar{f}^{l}(H, P)=f^{l}(H, P)-H^{j} P_{j}^{l}\right),
\end{aligned}
$$

and, completing the definition,

$$
G^{m+1}(X)=\left(H_{\infty}^{m+1}-X^{m+1}(y, t)\right) X_{y_{t}}^{i}+X^{m+1}\left[\delta_{i}^{\nu}-\left(\left(\underline{T}_{t}^{X}\right)^{-1}\right)_{x_{t}}^{\nu}\right] X_{y_{\nu}}^{i} .
$$

Now we estimate $G(X)$ in terms of $X$.

2.3. Lemma. For $T>0$ there is a $\delta_{3}>0$ and a constant $K_{4}(T)<$ $\infty$ such that if $X \in \mathscr{S}(T), \pi_{n} X \mid \partial \Omega=0$, and $\left\|X-H_{\infty}\right\|_{\mathscr{S}(T)} \leq \delta_{2}$ then $G(X) \in C^{0}\left((0, T],\left(H_{p}^{1}\right)^{m} \times H_{p}^{2}\right)$, and

$$
\begin{aligned}
& \|G\|_{C^{0}\left([t, T],\left(H_{p}^{1}\right)^{m} \times H_{p}^{2}\right)}+\|G\|_{C^{1 / 4}\left([t, T],\left(L_{p}\right)^{m} \times H_{p}^{1}\right)} \\
& \quad \leq t^{-1 / 2} K_{4}(T)\left(\left\|X-H_{\infty}\right\|_{\mathscr{S}(T)}^{2}+\|(0, g)\|_{\mathscr{B}}\right) .
\end{aligned}
$$

Proof. This is an easy consequence of Lemma 3.5 of [11].

2.4. LemMA. The operator $-A$ generates an analytic semigroup on $B_{p}$, and there is a number $K_{5}<+\infty$ such that for $X \in B_{p}$ we have

$$
\begin{gathered}
\left\|e^{-t A} X\right\|_{B_{p}} \leq K_{5} e^{-\eta t}\|X\|_{B_{p}}, \\
\left\|e^{-t A} X\right\|_{D_{A}^{p}} \leq K_{5}\left(1+t^{-1}\right) e^{-\eta t}\|X\|_{B_{p}},
\end{gathered}
$$

and if $X$ also belongs to $\left(H_{p}^{1}\right)^{m} \times H_{p}^{2}$ the additional inequalities

$$
\begin{gathered}
\left\|e^{-t A} X\right\|_{\left(H_{p}^{1}\right)^{m} \times H_{p}^{2}} \leq K_{5}\left(1+t^{-1 / 2}\right) e^{-\eta t}\|X\|_{\left(H_{p}^{1}\right)^{m} \times H_{p}^{2}}, \\
\left\|e^{-t A} X\right\|_{\left(H_{p}^{3}\right)^{m} \times H_{p}^{2}} \leq K_{5}\left(1+t^{-3 / 2}\right) e^{-\eta t}\|X\|_{\left(H_{p}^{1}\right)^{m} \times H_{p}^{2}}, \\
\left\|e^{-t A} X\right\|_{H_{p}^{2}} \leq K_{5} e^{-\eta t}\left(\|X\|_{B_{p}}\left(1+t^{-1}\right)+\|X\|_{\left(H_{p}^{1}\right)^{m} \times H_{p}^{2}}\left(1+t^{-1 / 2}\right)\right), \\
\left\|e^{-t A} X\right\|_{H_{p}^{2}} \leq K_{5} e^{-\eta t}\left(1+t^{-1}\right)\|X\|_{H_{p}^{2}}\left(X \in H_{p}^{2}\right)
\end{gathered}
$$

are valid. 
Proof. The inequalities (1)-(4) are easily obtained from Condition C using the integral representation from p. 103 in [3] for $A-\eta I$. These then imply (5) as

$$
\begin{gathered}
\left\|\pi_{m} e^{-t A} X\right\|_{H_{p}^{2}} \leq C_{1}\left(t^{-1}+1\right) e^{-\eta t}\|X\|_{B_{p}} \quad \text { and } \\
\left\|\left(e^{-t A} X\right)^{m+1}\right\|_{H_{p}^{2}} \leq C_{2}\left(t^{-1 / 2}+1\right) e^{-\eta t}\|X\|_{\left(H_{p}^{1}\right)^{m} \times H_{p}^{2}} ;
\end{gathered}
$$

(6) is just a weakening of (5).

2.5. LEMMA. If $X(y, t) \in \mathscr{S}(T)$ is a solution of (E) in Lagrange coordinates on $[0, T]$, if $\phi \in C^{1}\left([0, \infty), H_{p}^{3}\right) \cap C^{2}\left([0, \infty), H_{p}^{1}\right)$, $\phi^{\nu} \equiv 0$ for $\nu \in\{1, \ldots, n, m+1\}$, and $\pi_{m} \phi\left|\partial \Omega=\pi_{m} X\right| \partial \Omega$, then for $t \in[0, T]$ we have $X_{t}+A_{1} X=G(X)$ and

$$
\begin{aligned}
X(y, t)= & M(X(y, t))+\phi(y, t) \\
& +e^{-t A}(X(y, 0)-\phi(y, 0)-M(X(y, 0))) \\
& +\int_{0}^{t} e^{-(t-s) A}\left(G(X)-M(G(X))-\phi_{t}-A_{1} \phi\right) d s .
\end{aligned}
$$

Proof. Using Theorem 2.7 of [11], we see that $X(y, t)$ fulfills the equation $X_{t}+A_{1} X=G(X)$; but $X$ does not usually even belong to $B_{p}$. However, $X-\phi-M(X-\phi) \in D_{A}^{p}$ for $t>0$ and

$$
\begin{aligned}
& (X-\phi-M(X-\phi))_{t}+A(X-\phi-M(X-\phi)) \\
& \quad=G(X)-M\left(X_{t}-\phi_{t}\right)-A_{1} M(X-\phi)-A_{1} \phi-\phi_{t} .
\end{aligned}
$$

Now $M \phi=0$ as $\phi^{m+1} \equiv 0, A_{1} M X=0$ because $f_{H^{m+1}}(H, 0)=0$, and

$$
|\Omega|\left(M A_{1} X\right)^{m+1}=H_{\infty}^{m+1} \int_{\Omega} \operatorname{div} \pi_{n} X d y=H_{\infty} \int_{\partial \Omega}\left(\pi_{n} X, n\right) d o=0,
$$

so $M X_{t}=-M A_{1} X+M G=M G$, and

$$
(X-\phi-M X)_{t}+A(X-\phi-M X)=G(X)-M G(X)-A_{1} \phi-\phi_{t} .
$$

As the right side of the above equation belongs to $C^{1 / 4}\left([\hat{\delta}, T], B_{p}\right)$ for all $\hat{\delta}>0$ we get the desired representation from Theorem 3.2 (p. 109) of [3] for the interval $[\hat{\delta}, T]$, and can then let $\hat{\delta}$ go to zero.

2.6. LemMA. There is a constant $K_{6}<+\infty$ such that if $h(t):(0, T]$ $\rightarrow B_{p}$ fulfills the inequality

$$
\|h\|_{C^{1 / 4}\left([t, T], B_{p}\right)}+\|h\|_{C^{0}\left([t, T],\left(H_{p}^{1}\right)^{m} \times H_{p}^{2}\right)} \leq \frac{\alpha}{\sqrt{t}} \quad(t>0),
$$


then for $t \geq 2$ we have

$$
\hat{h}(t)=\int_{0}^{t} e^{-(t-s) A} h(s) d s \in H_{p}^{2}
$$

and $\|\hat{h}(t)\|_{H_{p}^{2}} \leq K_{6} \alpha$.

Proof. We can write $\hat{h}(t)$ as the sum of the two functions

$$
h_{1}(t)=\int_{0}^{t} e^{-(t-s) A} d s h(t)
$$

and

$$
h_{2}(t)=\int_{0}^{t} e^{-(t-s) A}(h(s)-h(t)) d s
$$

As

$$
\frac{d}{d \tau}\left(A^{-1} e^{-\tau A} h(t)\right)=-e^{-\tau A} h(t)
$$

we have $h_{1}(t)=A^{-1} h(t)-A^{-1} e^{-t A} h(t)$; therefore

$$
\left\|h_{1}(t)\right\|_{H_{p}^{2}} \leq C_{1}\|h(t)\|_{\left(H_{p}^{1}\right)^{m} \times H_{p}^{2}} \leq C_{2} \alpha \quad(t \geq 2) .
$$

Using 2.4 we also obtain

$\left\|h_{2}(t)\right\|_{H_{p}^{2}}$

$$
\begin{aligned}
\leq & C_{3} \int_{0}^{t} e^{-\eta(t-s)}\left[\|h(s)-h(t)\|_{B_{p}}\left(|s-t|^{-1}+1\right)\right. \\
& \left.+\|h(s)-h(t)\|_{\left(H_{p}^{1}\right)^{m} \times H_{p}^{2}}\left(|s-t|^{-1 / 2}+1\right)\right] d s \\
\leq & C_{4} \int_{0}^{t / 2} e^{-\eta(t-s)}\left[\|h(s)\|_{\left(H_{p}^{1}\right)^{m} \times H_{p}^{2}}+\|h(t)\|_{\left(H_{p}^{1}\right)^{m} \times H_{p}^{2}}\right] d s \\
& +C_{5} \alpha \int_{t / 2}^{t} e^{-\eta(t-s)}\left(|s-t|^{-3 / 4}+1\right) d s \\
\leq & C_{6} \alpha \int_{0}^{t / 2} e^{-\eta(t-s)}\left(1+s^{-1 / 2}\right) d s \\
& +C_{7} \alpha \int_{t / 2}^{t} e^{-\eta(t-s)}\left(1+|s-t|^{-3 / 4}\right) d s
\end{aligned}
$$

$\leq C_{8} \alpha$ for $t \geq 2$.

This immediately implies our assertion.

2.7. LEMMA. For every $T>0$ there are constants $K_{7}, \widetilde{K}_{7}(T)$, and $a \delta_{5} \in\left(0, \delta_{1}\right)$ such that if $X_{0}, \beta, g$ fulfill the conditions of 2.1 and

$$
\max \left(\left\|X_{0}-H_{\infty}\right\|_{H_{p}^{2}},\left\|\left(\beta-\pi_{m} H_{\infty}, g\right)\right\|\right)=\alpha \leq \delta_{5}
$$


then for the solution $X$ of $(\mathrm{E})$ corresponding to $X_{0}, \beta, g$ proven to exist in 2.1 we have the inequality

$$
\begin{aligned}
& \left\|X(x, t)-\widetilde{H}_{\infty}\left(X_{0}\right)\right\|_{H_{p}^{2}} \\
& \leq K_{7}\left(1+\widetilde{K}_{7}(T) \alpha\right) \\
& \quad \times\left[e^{-\eta t}\left\|X(x, 0)-\widetilde{H}_{\infty}\left(X_{0}\right)\right\|_{H_{p}^{2}}\right. \\
& \left.\quad+\widetilde{K}_{7}(T)\left(\left\|\left(\beta-\pi_{m} H_{\infty}, g\right)\right\|_{\mathscr{B}}+\left\|X_{0}-H_{\infty}\right\|_{H_{p}^{2}}^{2}\right)\right] \\
& \quad(t \in[2, T]) .
\end{aligned}
$$

Proof. First we choose $\delta_{5}>0$ so small that the solution obtained in 2.1 can be transformed into Lagrange coordinates over the whole interval $[0, T]$ by means of an admissible family of transformations $\underline{T}_{t}(y)$. Let $X(y, t)$ be this solution written in Lagrange coordinates. Note that there is a constant $C_{1}$ depending solely on the geometry of $\Omega$ such that a $\phi \in C^{1}\left([0, \infty), H_{p}^{3}\right) \cap C^{2}\left([0, \infty), H_{p}^{1}\right)$ can be chosen with $\pi_{m} \phi \mid \partial \Omega=\beta, \phi^{m+1} \equiv 0$, and

$$
\left\|\pi_{m}\left(\phi-H_{\infty}\right)\right\|_{H_{p}^{3}}+\left\|\phi_{t}\right\|_{H_{p}^{3}}+\left\|\phi_{t t}\right\|_{H_{p}^{1}} \leq C_{1}\left\|\left(\beta-\pi_{m} H_{\infty}, 0\right)\right\|_{\mathscr{B}} .
$$

Using 2.3, 2.4, 2.5, and 2.6, we get

$$
\begin{aligned}
\left\|X(y, t)-\widetilde{H}_{\infty}(X(y, t))\right\|_{H_{p}^{2}} & \\
\leq & C_{2} e^{-\eta t}\left(\left\|X(x, 0)-\widetilde{H}_{\infty}\left(X_{0}\right)\right\|_{H_{p}^{2}}+\left\|\pi_{m}\left(\phi(x, 0)-H_{\infty}\right)\right\|_{H_{p}^{2}}\right) \\
& +\left\|\pi_{m}\left(\phi(y, t)-H_{\infty}\right)\right\|_{H_{p}^{2}} \\
& \quad+\left\|\int_{0}^{t} e^{-(t-s) A}\left(G(X)-M G(X)-\phi_{t}-A_{1} \phi\right) d s\right\|_{H_{p}^{2}} \\
\leq & C_{2} e^{-\eta t}\left\|X(x, 0)-\widetilde{H}_{\infty}\left(X_{0}\right)\right\|_{H_{p}^{2}}+C_{3}(T)\left\|\left(\beta-\pi_{m} H_{\infty}, g\right)\right\|_{\mathscr{B}} \\
& +C_{4}(T)\left\|X-H_{\infty}\right\|_{\mathscr{S}(T)}^{2} .
\end{aligned}
$$

As $X(x, t)=X\left(\underline{T}_{t}^{-1}(x), t\right)$ we also get

$$
\begin{aligned}
& \left\|X(x, t)-\widetilde{H}_{\infty}(X(y, t))\right\|_{H_{p}^{2}} \\
& \quad \leq\left(1+C_{5}(T) \alpha\right)\left\|X(y, t)-\widetilde{H}_{\infty}(X(y, t))\right\|_{H_{p}^{2}}
\end{aligned}
$$


and, using the time-independence of $\widetilde{H}_{\infty}(X(x, t))$,

$$
\begin{aligned}
|\Omega| \cdot \mid & \left|\widetilde{H}_{\infty}(X(y, t))-\widetilde{H}_{\infty}\left(X_{0}\right)\right| \\
& =\left|\int_{\Omega} X^{m+1}(y, t)-H_{\infty}^{m+1} d y-\int_{\Omega} X^{m+1}(x, t)-H_{\infty}^{m+1} d x\right| \\
& =\left|\int_{\Omega}\left(X^{m+1}(y, t)-H_{\infty}^{m+1}\right)\left(1-J_{\underline{T} t}(y)\right) d y\right| \\
& \leq C_{6}(T)\left\|X^{m+1}(y, t)-H_{\infty}\right\|_{L_{\infty}} \cdot \int_{0}^{T}\left\|\nabla \pi_{m} X\right\|_{L_{\infty}} d \tau \\
& \leq C_{7}(T)\left\|X(y, t)-H_{\infty}\right\|_{\mathscr{S}(T)}^{2} .
\end{aligned}
$$

By 2.1 we have

$$
\begin{aligned}
\| X- & H_{\infty} \|_{\mathscr{S}(T)} \\
& \leq C_{8}(T)\left(\left\|X(x, 0)-H_{\infty}\right\|_{H_{p}^{2}}+\left\|\left(\beta-\pi_{m} H_{\infty}, g\right)\right\|_{\mathscr{B}}\right),
\end{aligned}
$$

which implies our claim.

Now we can prove Theorem 1.1.

First we choose $T \geq 2$ large enough so that $K_{7} e^{-\eta T} \leq \frac{1}{4}$. Once this is done, we need no longer indicate the dependence of our constants on $T$. With $\alpha_{1}=\min \left(\delta_{5},\left(4 \widetilde{K}_{7}\right)^{-1},\left(K_{7} \widetilde{K}_{7} 8\right)^{-1}\right)$ we obtain from Lemma 2.7 that if

$$
\max \left(\left\|X_{0}-H_{\infty}\right\|_{H_{p}^{2}},\left\|\left(\beta-\pi_{m} H_{\infty}, g\right)\right\|_{1}\right) \leq \alpha_{1},
$$

then there is a solution $X$ of $(\mathrm{E})$ on $[0, T]$ fulfilling the inequality

$$
\begin{aligned}
&\left\|X(x, T)-\widetilde{H}_{\infty}\left(X_{0}\right)\right\|_{H_{p}^{2}} \\
& \leq \frac{1}{3}\left\|X(x, 0)-\widetilde{H}_{\infty}\left(X_{0}\right)\right\|_{H_{p}^{2}} \\
&+C_{1}\left(\left\|\left(\beta-\pi_{m} H_{\infty}, g\right)\right\|_{\mathscr{B}}+\left|H_{\infty}-\widetilde{H}_{\infty}\left(X_{0}\right)\right|\right) \\
&+K_{7} \widetilde{K}_{7} \frac{5}{4} \alpha_{1}\left\|X(x, 0)-\widetilde{H}_{\infty}\left(X_{0}\right)\right\|_{H_{p}^{2}} \\
& \leq \frac{1}{2}\left\|X(x, 0)-\widetilde{H}_{\infty}\left(X_{0}\right)\right\|_{H_{p}^{2}} \\
&+C_{1}\left(\left\|\left(\beta-\pi_{m} H_{\infty}, g\right)\right\|_{\mathscr{B}}+\left|H_{\infty}-\widetilde{H}_{\infty}\left(X_{0}\right)\right|\right) .
\end{aligned}
$$

This immediately gives us

$$
\begin{aligned}
\left\|X(x, T)-H_{\infty}\right\|_{H_{p}^{2}} \leq & \frac{1}{2}\left\|X(x, 0)-H_{\infty}\right\|_{H_{p}^{2}} \\
& +C_{2}\left(\left\|\left(\beta-\pi_{m} H_{\infty}, g\right)\right\|_{1}+\left|H_{\infty}-\widetilde{H}_{\infty}\left(X_{0}\right)\right|\right) .
\end{aligned}
$$


If we choose $\varepsilon_{1}$ small enough to assure

$$
C_{2}\left(\left\|\left(\beta-\pi_{m} H_{\infty}, g\right)\right\|_{1}+\left|H_{\infty}-\tilde{H}_{\infty}\left(X_{0}\right)\right|\right) \leq \alpha_{1} / 2,
$$

then $\left\|X(x, 0)-H_{\infty}\right\|_{H_{p}^{2}} \leq \alpha_{1}$ implies $\left\|X(x, T)-H_{\infty}\right\|_{H_{p}^{2}} \leq \alpha_{1}$. So we can continue our solution to $[0,2 T]$. As $\widetilde{H}_{\infty}(X(x, t))$ is constant and $\left\|\left(\beta-\pi_{m} H_{\infty}, g\right)\right\|_{\mathscr{B}}$ cannot be larger for these new initial value problems than it was for the original one, this can be repeated indefinitely.

3. Asymptotic behavior. In order to prove Theorem 1.2 we consider solutions $\widetilde{X}_{1}$ and $\widetilde{X}_{2}$ of (E) with right sides $\tilde{g}_{1}, \tilde{g}_{2}$; and assume these, and the initial and boundary values fulfill the conditions of Theorem 1.1. We define $X_{i}(x, t)=\widetilde{X}_{i}(x, t+1), \beta_{i}=X_{i} \mid \partial \Omega$, and $g_{i}(x, t)=\tilde{g}_{i}(x, t+1)(x \in \bar{\Omega}, t \geq 0, i=1,2)$. We shall derive some estimates for $Z(x, t)=X_{1}(x, t)-X_{2}(x, t)$ assuming $M\left(X_{1}(x, t)\right)=$ $M\left(X_{2}(x, t)\right)$; i.e., $M(Z(x, t))=0$. With

$$
\omega=\sum_{i=1}^{2}\left(\left\|\widetilde{X}_{i}(x, 0)-H_{\infty}\right\|_{H_{p}^{2}}+\left\|\left(\pi_{m}\left(\tilde{X}_{i} \mid \partial \Omega-H_{\infty}\right), \tilde{g}_{i}\right)\right\|_{\mathscr{B}}\right)
$$

then we have

$$
\sum_{i=1}^{2}\left(\left\|X_{i}(x, t)\right\|_{H_{p}^{2}}+\left\|\pi_{m} X_{i}(x, t)\right\|_{H_{p}^{3}}\right) \leq K_{1} \omega
$$

by Theorem 1.1. For given $T<+\infty$ the transformation of any function into Lagrange coordinates with respect to $X_{1}$ on the interval $[0, T]$ is therefore possible by means of an admissible family of transformations $x=\underline{T}_{t}(y)$, if $\omega$ is small enough. In what follows we always assume this to be the case. Then we have

3.1. LEMMA. The transformation $Z(y, t)$ of $Z(x, t)$ into Lagrange coordinates with respect to $X_{1}$ fulfills the equation

$$
\begin{gathered}
Z_{t}(y, t)+\tilde{A}_{1}(t) Z(y, t)=\gamma(y, t)+B(t) Z(y, t) \\
\left(\gamma=\left(g_{1}^{1}-g_{2}^{1}, \ldots, g_{1}^{m}-g_{2}^{m}, 0\right)\right) \text { on }[0, T] \text { with linear operators } \\
\widetilde{A_{1}}(t):\left(H_{p}^{2}\right)^{m} \times H_{p}^{1} \rightarrow\left(L_{p}\right)^{m} \times H_{p}^{1} \text { and } \\
B(t):\left(H_{p}^{2}\right)^{m} \times H_{p}^{1} \rightarrow\left(L_{p}\right)^{m} \times H_{p}^{1}
\end{gathered}
$$


having the property that

$$
\begin{gathered}
B(t) h \in C^{0}\left([0, T],\left(L_{p}\right)^{m} \times H_{p}^{1}\right) \\
\left\|\left(\widetilde{A_{1}}(t)-\widetilde{A}_{1}(s)\right) h\right\|_{B_{p}} \leq K_{8}(T) \omega|t-s|^{1 / 2}\|h\|_{\left(H_{p}^{2}\right)^{m} \times H_{p}^{1}}, \\
\left\|\left(\widetilde{A_{1}}(t)-A_{1}\right) h\right\|_{B_{p}} \leq K_{8}(T) \omega\|h\|_{\left(H_{p}^{2}\right)^{m} \times H_{p}^{1}}, \\
\|B(t) h\|_{\left(L_{p}\right)^{m} \times H_{p}^{1}} \leq K_{8}(T) \omega\|h\|_{H_{p}^{1}}
\end{gathered}
$$

for $t, s \in[0,1], h \in\left(H_{p}^{2}\right)^{m} \times H_{p}^{1}$ with $\pi_{n} h \mid \partial \Omega=0$.

Proof. In the Euler coordinates we have for $l=1, \ldots, m$,

$$
Z_{t}^{l}+f^{l}\left(X_{1}, \nabla X_{1}\right)-f^{l}\left(X_{2}, \nabla X_{2}\right)=L_{X_{1}}^{l} X_{1}-L_{X_{2}}^{l} X_{2}+\gamma^{l} .
$$

With $X_{\tau}=(2-\tau) X_{1}+(\tau-1) X_{2}$ we then obtain

$$
\begin{aligned}
Z_{t}^{l}+ & \int_{1}^{2} f_{H^{i}}^{l}\left(X_{\tau}, \nabla X_{\tau}\right) d \tau Z^{i}+\sum_{\nu=1}^{n} \sum_{\mu=1}^{m} \int_{1}^{2} f_{P_{\nu}^{\mu}}^{l}\left(X_{\tau}, \nabla X_{\tau}\right) d \tau Z_{x_{\nu}}^{\mu} \\
& =L_{X_{1}}^{l} Z+\int_{1}^{2}\left(a_{k}^{\lambda \mu l}\right)_{H^{\nu}}\left(X_{\tau}\right) d \tau Z^{\nu}\left(X_{2}^{k}\right)_{x_{\lambda} x_{\mu}}+\gamma^{l} .
\end{aligned}
$$

Transforming this into Lagrange coordinates we see that

$$
\begin{aligned}
\widetilde{A}_{1}^{l}(t) Z= & f_{H_{i}}^{l}\left(H_{\infty}, 0\right) Z^{i}+\sum_{\nu=1}^{n} \sum_{\mu=1}^{m} f_{P_{\nu}^{\mu}}^{l}\left(H_{\infty}, 0\right) Z_{y_{\nu}}^{\mu} \\
& -a_{\lambda}^{k \mu l}\left(X_{1}\right)\left(\underline{T}_{t}^{-1}\right)_{x_{k}}^{\nu}\left(\underline{T}_{t}^{-1}\right)_{x_{\mu}}^{\pi} Z_{y_{\nu} y_{\pi}}^{\lambda},
\end{aligned}
$$

and the $B^{l}(t)$ determined thereby for $l=1, \ldots, m$ fulfill our conditions. For the $(m+1)$ st component we have in Euler coordinates

$$
X_{i t}^{m+1}+\left(X_{i}^{m+1} X_{i}^{j}\right)_{x_{j}}=0 \quad(i=1,2)
$$

which implies

$$
Z_{t}^{m+1}+X_{1}^{j} Z_{x_{j}}^{m+1}+Z^{m+1} X_{1 x_{j}}^{j}+X_{2 x_{j}}^{m+1} Z^{j}+X_{2}^{m+1} Z_{X_{j}}^{j}=0
$$

so in Lagrange coordinates with respect to $X_{1}$ we get

$$
\begin{aligned}
0= & Z_{t}^{m+1}(y, t)+Z^{m+1}\left(\underline{T}_{t}^{-1}\right)_{x_{\nu}}^{\mu} X_{1 y_{\mu}}^{\nu} \\
& +X_{2 y_{\nu}}^{m+1}\left(\underline{T}_{t}^{-1}\right)_{x_{j}}^{\nu} Z^{j}+X_{2}^{m+1} Z_{y_{\nu}}^{j}\left(\underline{T}_{t}^{-1}\right)_{x_{j}}^{\nu}
\end{aligned}
$$

With

$$
\widetilde{A}^{m+1}(t) Z=X_{2}^{m+1} Z_{y_{\nu}}^{j}\left(\underline{T}_{t}^{-1}\right)_{x_{\jmath}}^{\nu}-M\left(\left(0, \ldots, 0, X_{2}^{m+1} Z_{y_{\nu}}^{j}\left(\underline{T}_{t}^{-1}\right)_{x_{\jmath}}^{\nu}\right)\right)
$$


and the $B^{m+1}(t)$ thus determined our claims are easily verified as $H_{\infty}^{m+1} \int_{\Omega} Z_{y_{\nu}}^{\nu} d y=0$ by Gauss's theorem, and therefore

$$
\left\|M\left(0, \ldots, 0, X_{2}^{m+1} Z_{y_{\nu}}^{j}\left(\underline{T}_{t}^{-1}\right)_{x_{j}}^{\nu}\right)\right\|_{L_{p}} \leq C_{2}(T) \omega\|Z\|_{H_{p}^{1}}
$$

3.2. LemMA. There is a constant $K_{9}<+\infty$ such that to every $T>0$ there exists a $\delta_{6}>0$ having the following property: If $\omega \leq \delta_{6}$, then the fundamental solution $\Gamma(t, s)$ on $B_{p}$ (see [3], part 2, chap. 4-6) of the equation

$$
h_{t}+\widetilde{A}(t) h=0 \quad\left(\widetilde{A}(t)=\widetilde{A}_{1}(t) \mid D_{A}^{p}\right)
$$

exists for $t, s \in[0, T], t \geq s$, and we have the inequality

$$
\begin{aligned}
\|\Gamma(t, s) h\|_{H_{p}^{1}} \leq K_{9} e^{-\eta(t-s) / 2}\|h\|_{B_{p}} & \left(1+|t-s|^{-1 / 2}\right) \\
& \left(h \in B_{p} ; t, s \in[0, T], t \geq s\right) .
\end{aligned}
$$

Proof. From Theorem 3.1 (p. 109), Lemma 7.1 (p. 127), and Theorem 10.1 (p. 27) of [3] it is clear that the fundamental solution exists, and the estimate is valid for $t-s \leq 2$. Also we can obviously assume

$$
\left\|e^{-\tau \widetilde{A}(t)} h\right\|_{B_{p}} \leq C_{1} e^{-2 \eta \tau / 3}\|h\|_{B_{p}} \quad(\tau \in[0, \infty))
$$

with a suitable constant $C_{1}<+\infty$. As $\Gamma(t, s)=\Gamma(t, t-1) \Gamma(t-1, s)$ for $t-s \geq 1$ we only need to prove

$$
\|\Gamma(t, s) h\|_{B_{p}} \leq \widetilde{C}_{1} e^{-\eta \tau / 2}\|h\|_{B_{p}}
$$

which can be done using equation 4.8 (p. 111) of [3] and the method employed to obtain 13.15 on p. 154 of [3].

3.3. LemmA. There are numbers $K_{10}, \delta_{7}, T \in(0, \infty)$ such that $\omega \leq \delta_{7}$ implies the inequality

$$
\begin{aligned}
& \|Z(x, T)\|_{H_{p}^{1}} \\
& \leq \frac{1}{2}\|Z(x, 0)\|_{H_{p}^{1}} \\
& +K_{10} \max _{\tau \in[0, T]}\left[\left\|g_{1}(\tau)-g_{2}(\tau)\right\|_{L_{p}}+\left\|\beta_{1}(\tau)-\beta_{2}(\tau)\right\|_{H_{p}^{2}(\partial \Omega)}\right. \\
& \left.+\left\|\beta_{1 t}(\tau)-\beta_{2 t}(\tau)\right\|_{L_{p}(\partial \Omega)}\right] \text {. }
\end{aligned}
$$

Proof. We start out by deriving an estimate for fixed arbitrary $T$. There is a constant $C_{1}$ only depending on the geometry of $\Omega$ such 
that we can always find a $\phi \in C^{1}\left([0, \infty), H_{p}^{3}\right) \cap C^{2}\left([0, \infty), H_{p}^{1}\right)$ with the properties

$$
\|\phi\|_{H_{p}^{2}}+\left\|\phi_{t}\right\|_{L_{p}} \leq C_{1}\left(\left\|\beta_{1}-\beta_{2}\right\|_{H_{p}^{2}}+\left\|\beta_{1 t}-\beta_{2 t}\right\|_{L_{p}}\right),
$$

and $\pi_{m} \phi \mid \partial \Omega=\beta_{1}-\beta_{2}, \phi^{m+1} \equiv 0$. In Lagrange coordinates with respect to $X_{1}$ now $Z$ is easily seen to fulfill the equation

$$
\begin{aligned}
(Z-\phi & -M Z)_{t}+\widetilde{A}(t)(Z-\phi-M Z) \\
= & \gamma+B(t) Z-\widetilde{A_{1}}(t) \phi-\phi_{t} \\
& \quad+\left(A_{1}-\widetilde{A_{1}}(t)\right) M Z(y, t)-M B(t) Z,
\end{aligned}
$$

as $A M Z=M A_{1} Z=M \gamma=0$. This gives us the representation

$$
\begin{aligned}
Z(y, t)= & \phi(y, t)+M(Z(y, t))+\Gamma(t, 0)(Z(x, 0)-\phi(x, 0)) \\
+ & \int_{0}^{t} \Gamma(t, s)\left[\gamma+B(s) Z-M B(s) Z-\widetilde{A}_{1}(s) \phi-\phi_{t}\right. \\
& \left.+\left(A_{1}-\tilde{A}_{1}(s)\right) M Z(y, s)\right] d s,
\end{aligned}
$$

from which, using 3.2 , we can derive the inequality

$$
\begin{aligned}
\sqrt{t} e^{\eta t / 2} & \|Z(y, t)\|_{H_{p}^{1}} \\
\leq & \sqrt{t} e^{\eta t / 2}\|M Z(y, t)\|_{H_{p}^{1}}+2 K_{9}\|Z(x, 0)\|_{H_{p}^{1}} \\
& +C_{2}(T) \sup _{\tau \in[0, T]}\left(\|\gamma(\tau)\|_{L_{p}}+\left\|\phi_{t}(\tau)\right\|_{L_{p}}+\|\phi(\tau)\|_{H_{p}^{2}}\right) \\
& +C_{3}(T) \omega \sup _{\tau \in[0, T]}\left(\sqrt{t} e^{\eta \tau / 2}\|Z(y, t)\|_{H_{p}^{1}}\right) .
\end{aligned}
$$

As in the proof of Theorem 2.7 we see that

$$
|M(Z(y, t))| \leq C_{4}(T) \omega\|Z\|_{H_{p}^{1}},
$$

so taking the supremum on both sides of the inequality for $t \in[0, T]$ we get

$$
\begin{aligned}
\sup _{t \in[0, T]} & \left(\sqrt{t} e^{\eta t / 2}\|Z(y, t)\|_{H_{p}^{1}}\right) \\
\leq & C_{5}\|Z(x, 0)\|_{H_{p}^{1}} \\
& +C_{6}(T) \sup _{\tau \in[0, T]}\left(\|\gamma(t)\|_{L_{p}}+\left\|\phi_{t}(t)\right\|_{L_{p}}+\|\phi(t)\|_{H_{p}^{2}}\right)
\end{aligned}
$$

with a $C_{5}$ independent of $T$, provided $\omega$ is small enough. To obtain our statement we now choose $T$ so large that $e^{-\eta T / 2} C_{5} \frac{1}{\sqrt{T}} \leq \frac{1}{4}$, and 
then $\delta_{7}$ small enough to make the above estimate true for $\omega \leq \delta_{7}$ and in addition

$$
\|Z(x, T)\|_{H_{p}^{1}} \leq 2\|Z(y, T)\|_{H_{p}^{1}}, \quad\|\gamma(y, t)\|_{L_{p}} \leq 2\|\gamma(x, t)\|_{L_{p}}
$$

for $t \in[0, T]$.

Now we can prove 1.2 . First consider the solution $\tilde{X}(x, t)$ of the problem with $\beta(t)=\hat{\beta}, g(t)=\hat{g}$, and a suitable initial value, and let $\widetilde{X}_{1}=\widetilde{X}, \widetilde{X}_{2}(t)=\widetilde{X}(t+\Delta t)$ with a $\Delta t>0$. We choose $\varepsilon_{2}>0$ small enough to be able to apply 3.3 starting at any point $t \in[0, \infty)$ instead of 0 . This gives us

$$
\begin{array}{r}
\|\widetilde{X}(T+t+\Delta t)-\tilde{X}(T+t)\|_{H_{p}^{1}} \leq \frac{1}{2}\|\tilde{X}(t+\Delta t)-\tilde{X}(t)\|_{H_{p}^{1}} \\
(t \in[0, \infty)),
\end{array}
$$

as our equation is autonomous in this case. Dividing by $\Delta t$ and letting $\Delta t \rightarrow 0$ we get

$$
\left\|\widetilde{X}_{t}(T+t)\right\|_{H_{p}^{1}} \leq \frac{1}{2}\left\|\widetilde{X}_{t}(t)\right\|_{H_{p}^{1}}
$$

so $\widetilde{X}_{t}(t) \rightarrow 0$ in $H_{p}^{1}$ and $\int_{1}^{\infty}\left\|\widetilde{X}_{t}(t)\right\|_{H_{p}^{1}}<+\infty$.

So $\widetilde{X}$ converges in $H_{p}^{1}$, and its limit $\widehat{X}$ must belong to $H_{p}^{2}$ and $\pi_{m} \widehat{X} \in H_{p}^{3}$. As $\widehat{X}$ is a stationary solution of $(\mathrm{E})$, it is also unique by 3.3.

For arbitrary $\beta(t), g(t)$ we apply 3.3 to $\widetilde{X}_{1}=X, \widetilde{X}_{2}=\widehat{X}$, and obtain

$$
\begin{aligned}
&\|X(x, T+t)-\widehat{X}(x)\|_{H_{p}^{1}} \\
& \leq \frac{1}{2}\|X(x, t)-\widehat{X}(x)\|_{H_{p}^{1}} \\
&+K_{10} \sup _{\tau \in[t, t+t]}\left(\|g(\tau)-\hat{g}\|_{L_{p}}+\|\beta(\tau)-\hat{\beta}\|_{H_{p}^{2}}+\left\|\beta_{t}\right\|_{L_{p}}\right) .
\end{aligned}
$$

Taking the limsup on both sides this implies $X(x, t) \rightarrow \widehat{X}(x)$ in $H_{p}^{1}$, from which the remainder follows easily.

\section{REFERENCES}

[1] S. Agmon, A. Douglis, and L. Nirenberg, Estimates near the boundary for solutions of elliptic partial differential equations satisfying general boundary conditions, Comm. Pure Appl. Math., 27 (1964), 35-92.

[2] A. B. Cambel, Plasma Physics and Magneto-Fluid Mechanics, New York: McGraw Hill, 1963. 
[3] A. Friedman, Partial Differential Equations, New York: Holt, Rinehart, Winston, 1969.

[4] A. V. Kazhikov and V. V. Shelukhin, Unique global solution with respect to time of initial boundary value problems for one-dimensional equations of a viscous gas, J. Appl. Math. Mech., 41 (1977), 273-282.

[5] A. Matsumura and T. Nishida, The initial value problem for the equations of motion of compressible viscous and heat-conductive fluids, Proc. Japan Acad. Ser. A, 55 (1979), 337-342.

[6] _ Initial-boundary value problems for the equations of compressible viscous and heat-conductive fluid, Lecture Notes in Numerical and Applied Analysis, 5 (1982), 153-170, North Holland, Edited by H. Fujita, P. D. Lax, G. Strang,

[7] Initial-boundary value problems for the equations of motion of compressible viscous and heat-conductive fluids, Comm. Math. Phys., 89 (1983), 445-464.

[8] M. Padula, Existence of global solutions for 2-dimensional viscous compressible flows, J. Funct. Anal., 69 (1986), 1-20.

[9] J. A. Shercliff, A Textbook of Magnetohydrodynamics, Oxford: Pergamon Press, 1965.

[10] G. Ströhmer, About the resolvent of an operator from fluid dynamics, Math. Z., 194 (1987), 183-191.

[11] _ About a certain class of parabolic-hyperbolic systems of differential equations, Analysis, 9 (1989), 1-39.

[12] W. v.Wahl, The equations of Navier-Stokes and abstract parabolic equations, Braunschweig, F. Vieweg, 1985.

Received December 15, 1987.

UNIVERSITY OF IOWA

IOWA CITY, IA 52242 



\section{PACIFIC JOURNAL OF MATHEMATICS EDITORS}

\author{
V. S. VARADARAJAN \\ (Managing Editor) \\ University of California \\ Los Angeles, CA 90024-1555-05 \\ Herbert Clemens \\ University of Utah \\ Salt Lake City, UT 84112 \\ ThOMAs ENRIGHT \\ University of California, San Diego \\ La Jolla, CA 92093
}

R. FINN

Stanford University

Stanford, CA 94305

HeRmanN FlaschKa

University of Arizona

Tucson, AZ 85721

VAUGHAN F. R. JoNES

University of California

Berkeley, CA 94720

SteVen KerCKhofF

Stanford University

Stanford, CA 94305
ROBION KIRBY

University of California

Berkeley, CA 94720

C. C. Moore

University of California

Berkeley, CA 94720

HAROLD STARK

University of California, San Diego

La Jolla, CA 92093

\section{ASSOCIATE EDITORS}
R. ARENS
E. F. BECKENBACH
B. H. NeumanN
F. Wolf
K. YoshidA
(1906-1982)
(1904-1989)

\section{SUPPORTING INSTITUTIONS}

UNIVERSITY OF ARIZONA
UNIVERSITY OF BRITISH COLUMBIA
CALIFORNIA INSTITUTE OF TECHNOLOGY
UNIVERSITY OF CALIFORNIA
MONTANA STATE UNIVERSITY
UNIVERSITY OF NEVADA, RENO
NEW MEXICO STATE UNIVERSITY
OREGON STATE UNIVERSITY
UNIVERSITY OF ARIZONA
UNIVERSITY OF BRITISH COLUMBIA
UNIVERSITY OF CALIFORNIA
MONTANA STATE UNIVERSITY
NEW MEXICO STATE UNIVERSITY

\author{
UNIVERSITY OF OREGON \\ UNIVERSITY OF SOUTHERN CALIFORNIA \\ STANFORD UNIVERSITY \\ UNIVERSITY OF HAWAII \\ UNIVERSITY OF TOKYO \\ UNIVERSITY OF UTAH \\ WASHINGTON STATE UNIVERSITY \\ UNIVERSITY OF WASHINGTON
}

The Supporting Institutions listed above contribute to the cost of publication of this Journal, but they are not owners or publishers and have no responsibility for its content or policies.

Mathematical papers intended for publication in the Pacific Journal of Mathematics should be in typed form or offset-reproduced (not dittoed), double spaced with large margins. Please do not use built up fractions in the text of the manuscript. However, you may use them in the displayed equations. Underline Greek letters in red, German in green, and script in blue. The first paragraph must be capable of being used separately as a synopsis of the entire paper. In particular it should contain no bibliographic references. Please propose a heading for the odd numbered pages of less than 35 characters. Manuscripts, in triplicate, may be sent to any one of the editors. Please classify according to the 1980 Mathematics Subject Classification (1985 Revision) scheme which can be found in the December index volumes of Mathematical Reviews. Supply name and address of author to whom proofs should be sent. All other communications should be addressed to the managing editor, or Elaine Barth, University of California, Los Angeles, California 90024-1555-05.

There are page-charges associated with articles appearing in the Pacific Journal of Mathematics. These charges are expected to be paid by the author's University, Government Agency or Company. If the author or authors do not have access to such Institutional support these charges are waived. Single authors will receive 50 free reprints; joint authors will receive a total of 100 free reprints. Additional copies may be obtained at cost in multiples of 50 .

The Pacific Journal of Mathematics is issued monthly as of January 1966. Regular subscription rate: $\$ 190.00$ a year (6 Vols., 12 issues). Special rate: $\$ 95.00$ a year to individual members of supporting institutions.

Subscriptions, orders for numbers issued in the last three calendar years, and changes of address should be sent to Pacific Journal of Mathematics, P.O. Box 969, Carmel Valley, CA 93924, U.S.A. Old back numbers obtainable from Kraus Periodicals Co., Route 100, Millwood, NY 10546.

The Pacific Journal of Mathematics at P.O. Box 969, Carmel Valley, CA 93924 (ISSN 0030-8730) is published monthly. Second-class postage paid at Carmel Valley, California 93924, and additional mailing offices. Postmaster: send address changes to Pacific Journal of Mathematics, P.O. Box 969, Carmel Valley, CA 93924.

\section{PUBLISHED BY PACIFIC JOURNAL OF MATHEMATICS, A NON-PROFIT CORPORATION}




\section{Pacific Journal of Mathematics}

\section{Vol. 143, No. $2 \quad$ April, 1990}

Gustavo Corach, Horacio Porta and Lázaro Recht, Differential geometry of systems of projections in Banach algebras ................. 209

Peter Fleischmann and Jens Carsten Jantzen, Simple periodic modules of

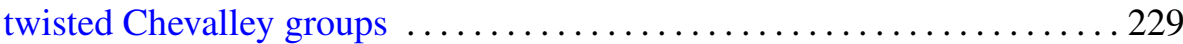

Niels Gronbaek, Amenability of discrete convolution algebras, the commutative case ...................................243

Nguyên H. V. Hung, The mod 2 equivariant cohomology algebras of

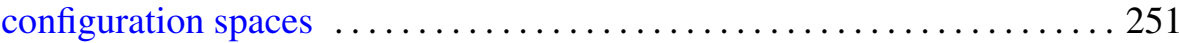

Wojciech Kucharz, Global almost analytic algebraicity of analytic sets . . . 287 John Merrill, A class of consistent anti-Martin's axioms .............. 301

Takafumi Murai, The power 3/2 appearing in the estimate of analytic

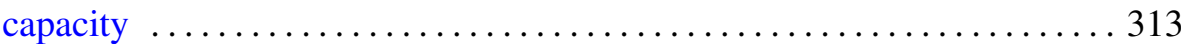

L. Panaitopol and Doru Stefanescu, On the generalized difference

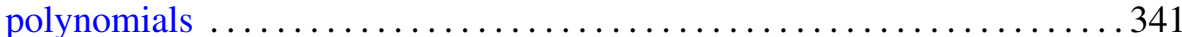

Katsuhiro Shiohama, Takashi Shioya and Minoru Tanaka, Mass of rays

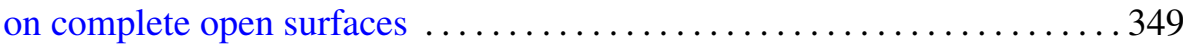

Gerhard Ströhmer, About compressible viscous fluid flow in a bounded

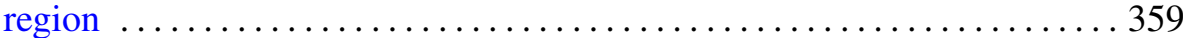

A. Ülger, Arens regularity sometimes implies the RNP 